\title{
Graph Self Supervised Learning: the BT, the HSIC, and the VICReg
}

\author{
Sayan $\mathrm{Nag}^{1}$ \\ ${ }^{1}$ nagsayan112358@gmail.com
}

May 29, 2021

\begin{abstract}
Self-supervised learning and pre-training strategies have developed over the last few years especially for Convolutional Neural Networks (CNNs). Recently application of such methods can also be noticed for Graph Neural Networks (GNNs). In this paper, we have used a graph based self-supervised learning strategy with different loss functions (Barlow Twins[7], HSIC[4], VICReg[1]) which have shown promising results when applied with CNNs previously. We have also proposed a hybrid loss function combining the advantages of VICReg and HSIC and called it as VICRegHSIC. The performance of these aforementioned methods have been compared when applied to two different datasets namely MUTAG and PROTEINS. Moreover, the impact of different batch sizes, projector dimensions and data augmentation strategies have also been explored. The results are preliminary and we will be continuing to explore with other datasets.
\end{abstract}

\section{Introduction}

A mainstream way of training a deep neural network is to feed some input in order to get certain desirable outputs. Such a training is better known as supervised learning in which a sufficient amount of input data and label pairs are fed to the model depending on its complexity. However, such a method calls for a large number of labels thereby rendering the supervised learning scheme practically inapplicable in many applications because of the unavailability of such large annotated datasets. Dataset labeling not only comes with huge overhead costs, but also can be biased depending on certain circumstances. Thus a need for a better learning strategy led to the advent and thereby adoption of self-supervised learning (SSL) which enables the training of models on unlabeled data. This removes the burden of labeling and therefore it is an efficient and lucrative approach. In the presence of a handful of labeled data, using SSL strategy representations from the remaining unlabeled data can be learnt. Now, the same can be used as a pre-training procedure after which labeled data are used to fine-tune the pre-trained models. These fine-tuned models can either be used for downstream tasks, or as an auxiliary training task that enhances the performance of main tasks. SSL has shown promising results in the field of computer visions using cnns [3] as well as with graph neural networks [5].

The primary contributions of this paper are:

1. We have used four different loss functions namely Barlow Twins Loss, HSIC Loss, VICReg Loss and proposed hybrid VICRegHSIC Loss in the Graph based SSL paradigm and have compared their perfomances on node classification tasks. To the best of our knowledge it is the first work where Graph SSL has been used with such loss functions.

2. We have also demonstrated the impacts of different batch sizes, data augmentations and projector dimensions on the aforementioned losses.

This work shows some preliminary analyses and results and more experiments will be done in the future for different datasets and tasks to provide a better understanding of GNNs for SSL.

\section{Related Work}

Self-supervised learning has gained a lot of momentum nowadays. It is being applied more in computer vision tasks [3]. Barlow Twins is one such self-supervised learning framework which tries to maximize the agreement between two learned representations of a pair of distorted inputs with a novel Barlow Twins loss function having an invariance term and a redundancy reduction term [7]. 


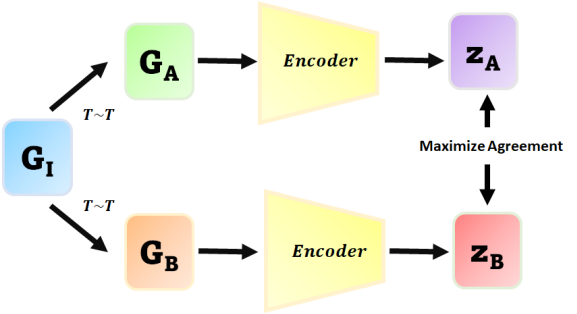

Figure 1: A standard paradigm of graph based self-supervised learning. Graph augmentations on input graph $G_{i}$ resulted in two transformed graphs $G_{A}$ and $G_{B}$ following each transformation $T$. A self-supervised loss is then computed between the encoded representations $z_{A}$ and $z_{B}$ in order to maximize the agreement.

Using Hilbert-Schmidt Independence Criterion, the authors in [4] introduced a modification to Barlow Twins Loss by altering the off-diagonal component of the empirical crosscorrelation matrix. Using such a negativesample-free contrastive approach, the authors claimed that the representations learnt will be superior. VICReg [1] uses the Barlow Twins' mechanism of decorrelation without any normalization in its loss function. Furthermore, it can prevent dimension collapse with the variance regularization term.

On the other hand contrastive methods like SIMCLR [2] use positive and negative sample pairs in their frameworks. More can be found in the survey paper [3]. Self-supervised learning has also been implemented for graph neural netorks. [5] provides an excellent review of such state-of-the-art methods. The field is rapidly advancing with promising results and we hope to witness more such advancements in the future.

\section{Methods}

A standard procedure for self-supervised learning for graphs includes graph data augmentation in order to create a pair of graphs, then finding the representations using a graph encoder followed by a projection head and finally using a loss function to maximize agreement between the pair of representations. The aforesaid paradigm has been represented in the Figure 1 where the projection head is not shown separately and can be considered to be existing in tandem with the encoder.

In our work we have followed the augmentations used by the authors in [6]. They are summarized in the table 1.

In our work we have used four different self-supervised loss functions which include the Barlow-Twin (BT) loss function as mentioned

\begin{tabular}{ll}
\hline Augmentation Methods & Type \\
\hline \hline Node Dropping (ND) & Nodes \\
Subgraph (SG) & Nodes, Edges \\
Edge Perturbation (EP) & Edges \\
Attribute Masking (AM) & Edges \\
\hline
\end{tabular}

Table 1: Data Augmentation methods for graphs.

in [7] (given by $L_{B T}$ ), the 2 Hilbert-Schmidt Independence Criterion (HSIC) loss function mentioned in [4] (given by $H S I C_{S S L}$ ), the VarianceInvariance-CovarianceRegularization (VICReg) loss function mentioned in [1], and finally a hybrid of the aforementioned VICReg loss and HSIC loss. In the hybrid loss we have just modifed the on- and off-diagonal terms of the covariance component of the VICReg as explained in [4].

\section{Experiments and Analysis}

For our work we have considered two datasets MUTAG and PROTEINS for node-classification tasks. This is an ongoing work and we will be extending it to other datasets and tasks. We have also done comparisons with respect to two different batch sizes and projector dimensions. This is a preliminary study and more comparisons will be done in the future to explore the impacts of the aforesaid factors on training with different self-supervised loss functions for graphs.

The Figure 2 shows that for MUTAG dataset increase in batch size for a projector dimension of 80 leads to a decrease in performance. However, increasing the projector dimension from 80 to 160 reverses the effect of increasing batch size and all except HSIC gives better performance. This is consistent with the performance of HSIC as demonstrated in [4]. For this analysis, we have considered data augmentations Node Drop and Subgraph together in the augmentation pool.

The Figure 3 shows that for MUTAG dataset increase in projector dimension for both the batch sizes leads to an increase in performance. It is more pronounced in batch size 64 and can be assumed that with even further increase in batch sizes the impact will be more. For this illustration, we have considered data augmentations Node Drop and Subgraph together in the augmentation pool.

The Figure 4 shows that for MUTAG dataset the performance of all the the methods drop (except for VICregHSIC) when edge peturbation 


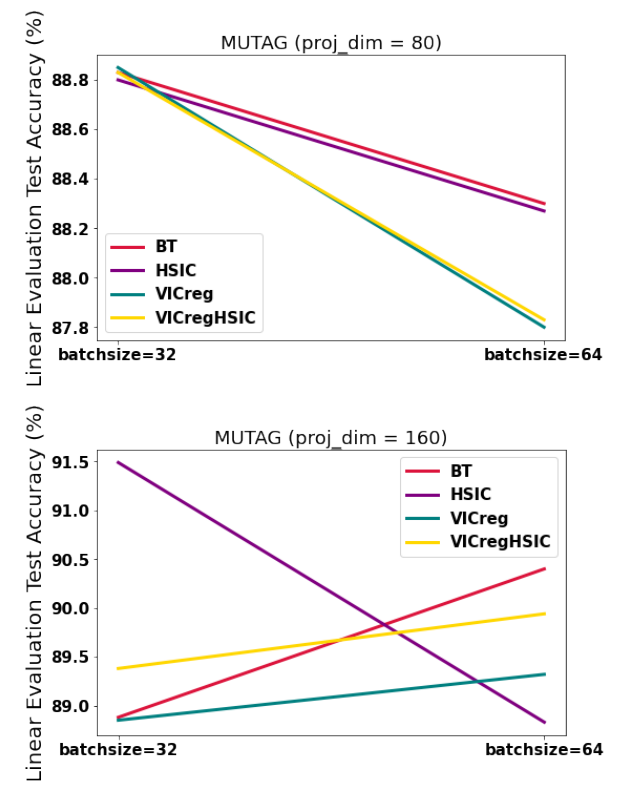

Figure 2: Impact of batch sizes on linear evaluation test accuracy

was added to node drop and subgraph. However, the performance increased again with addition of attribute masking to the previous subset. For VICregHSIC the performance kept on increasing with the addition of augmentations. This is an interesting observation and we do not have a decent explanation for this and we are investigating it at the moment.

The Figure 5 demonstrates the performance of different methods on the PROTEINS dataset when trained for 100 epochs. Faster convergence is observed for VICReg and VICRegHSIC. The training loss curves have been normalized for representation and comparison purposes.

The Figure 6 shows that for PROTEINS dataset the performance of all the the methods drop (except for VICregHSIC) when edge peturbation was added to node drop and subgraph. However, the performance increased again with addition of attribute masking to the previous subset. For VICregHSIC the performance kept on increasing with the addition of augmentations. This is similar to the one we had observed for MUTAG dataset (refer to Figure 4).

\section{$5 \quad$ Future Work}

Even though the results are interesting, this is a preliminary study and first of its kind with Graph Neural Networks and Barlow Twins and similar loss functions. More experiments need to be conducted to better understand the performance of these algorithms. More comparisons need to be done with other methods abreast

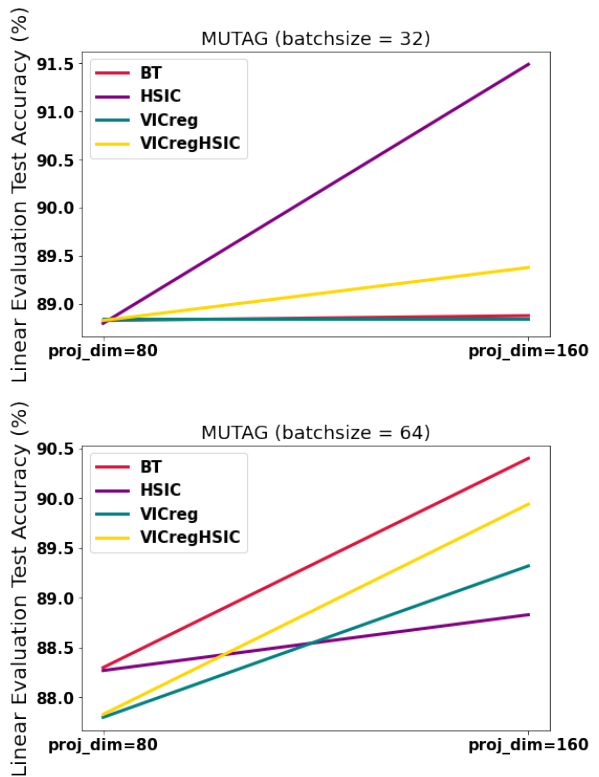

Figure 3: Impact of projector dimensions on linear evaluation test accuracy

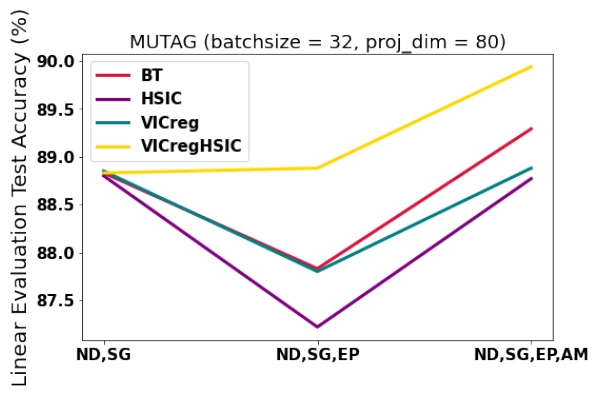

Figure 4: Impact of different graph data augmentations on linear evaluation test accuracy

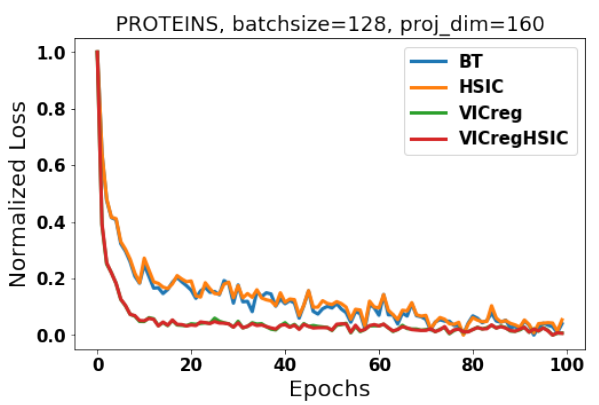

Figure 5: Normalized training loss curves (for representation purposes) for 100 epochs showing performance of different methods on PROTEINS dataset.

including more ablation studies. The training epochs also need to be increased to obtain improved performance and understand the results better. 


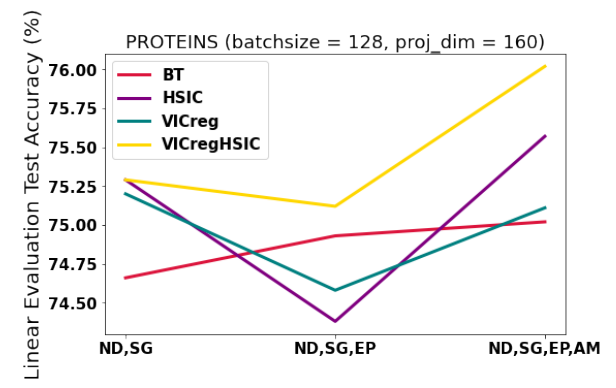

Figure 6: Impact of different graph data augmentations on linear evaluation test accuracy

\section{References}

[1] A. Bardes, J. Ponce, and Y. LeCun. Vicreg: Variance-invariance-covariance regularization for self-supervised learning. arXiv preprint arXiv:2105.04906, 2021.

[2] T. Chen, S. Kornblith, M. Norouzi, and G. Hinton. A simple framework for contrastive learning of visual representations. In International conference on machine learning, pages 1597-1607. PMLR, 2020.

[3] A. Jaiswal, A. R. Babu, M. Z. Zadeh, D. Banerjee, and F. Makedon. A survey on contrastive self-supervised learning. Technologies, 9(1):2, 2021.

[4] Y.-H. H. Tsai, S. Bai, L.-P. Morency, and R. Salakhutdinov. A note on connecting barlow twins with negative-samplefree contrastive learning. arXiv preprint arXiv:2104.13712, 2021.

[5] Y. Xie, Z. Xu, J. Zhang, Z. Wang, and S. Ji. Self-supervised learning of graph neural networks: A unified review. arXiv preprint arXiv:2102.10757, 2021.

[6] Y. You, T. Chen, Y. Sui, T. Chen, Z. Wang, and Y. Shen. Graph contrastive learning with augmentations. Advances in Neural Information Processing Systems, 33, 2020.

[7] J. Zbontar, L. Jing, I. Misra, Y. LeCun, and S. Deny. Barlow twins: Self-supervised learning via redundancy reduction. arXiv preprint arXiv:2103.03230, 2021. 